\title{
NOUVELLe
}

\section{Ki67 : un surfactant des chromosomes mitotiques}

Olivier Albagli ${ }^{1}$, Hélène Pelczar ${ }^{2}$
${ }^{1}$ CNRS-UMR 8014, Inserm U1016, groupe hospitalier Cochin-Port Royal, bâtiment Cassini, 123 boulevard de Port-Royal, 75014 Paris, France.

${ }^{2}$ Sorbonne Université, UFR 927, BC 60, 4 place Jussieu, 75252 Paris Cedex 05, France. olivier.albagli-curiel@inserm.fr helene.pelczar@upmc.fr
> L'antigène Ki67 a été identifié en 1983 par la recherche d'anticorps capables de reconnaître des protéines nucléaires de lymphome de Hodgkin [1]. Dès le début, il est apparu que, si cet antigène n'était pas spécifique de ces tumeurs, il n'était en revanche détectable que dans les cellules en train de proliférer (i.e. en cycle, hors phase $\mathrm{GO}$ ), que celles-ci soient normales ou tumorales, aussi bien in vivo que in vitro [1]. Cette caractéristique a été très largement confirmée et utilisée depuis, et l'expression du gène MKI67 (au niveau protéique ou, plus rarement, ARNm) est devenue un marqueur de prolifération couramment utilisé pour des types cellulaires très variés chez l'homme ou les rongeurs, en recherche fondamentale ou clinique, ou à des fins diagnostiques ou pronostiques en cancérologie [2, 3].

Pourtant, malgré son succès en tant que marqueur, la protéine Ki67, dont la séquence est connue depuis 1993, demeurait assez peu étudiée et sa fonction largement incomprise [2]. La grande taille des deux principales isoformes codées par le gène MKI67 (3256 et 2896 résidus chez l'hommel) et leur sensibilité à la dégradation par les protéases compliquaient les analyses biochimiques et moléculaires [3]. Ces difficultés laissaient en suspens une question essentielle: pourquoi la présence de Ki67 est-elle strictement associée à la prolifération cellulaire? Plusieurs observations déjà anciennes indiquaient que Ki67 est un constituant de la périphérie chromosomique,

${ }^{1}$ Deux ARNm, qui diffèrent par la présence ou l'absence d'un exon codant (exon 7), sont produits par épissage alternatif. une structure correspondant à environ un tiers du volume des chromosomes mitotiques $[2,4]$. Cependant, le rôle de cette structure observée depuis 130 ans demeurait assez énigmatique.

Une équipe a récemment élucidé un des rôles de Ki67 dans la périphérie chromosomique. Son travail a consisté à identifier des gènes impliqués dans la dispersion des chromosomes mitotiques [5]. Dans des cellules en mitose traitées par le nocodazole, qui empêche la formation du fuseau mitotique et bloque les cellules en prométaphase, les chromosomes se répartissent, probablement par diffusion aléatoire, dans tout le cytoplasme (l'enveloppe nucléaire n'existe plus à ce stade) (Figure 1). Cette dispersion en absence de fuseau mitotique est en réalité surprenante puisque les interactions produisant la condensation des chromosomes devraient conduire à leur regroupement [7]. Les chercheurs de cette équipe ont transfecté dans des cellules HeLa des ARN interférents (siARN) dirigés contre les ARNm codant 1295 protéines associées à la mitose, dont 67 codant des protéines de la périphérie chromosomique. Un seul de ces SiARN provoque le regroupement (coalescence ${ }^{2}$ ) des chromosomes, celui ciblant I'ARNm MKI67 [5]. L'implication spécifique de Ki67 dans la dispersion des chromosomes est confirmée par le fait que plusieurs siARN distincts ciblant I'ARNm MKI67 produisent le même phénotype [5]. De plus, la

\footnotetext{
${ }^{2}$ Les auteurs utilisent les mots agrégation [2], enchevêtrement [4], regroupement [5], collage [5], ou coalescence [5, $7,9]$. Tous ces termes décrivent la proximité anormale entre
} les chromosomes mitotiques.

modification, par la technique CRISPRCas9 [8] $(\rightarrow)$ de la séquence cible de I'un de ces siRnA abolit l'effet de ce siARN, mais pas celui des autres

$\rightarrow$ Voir la Synthèse de J.P. Tremblay, $\mathrm{m} / \mathrm{s}$ $n^{\circ} 11$, novembre 2015, page 1014 siARN ciblant des régions différentes de I'ARNm MKI67 [5]. Enfin, I'invalidation de MKI67 (par la technique CRISPR(as9) induit également la coalescence des chromosomes mitotiques dans les cellules HeLa [5] (Figure 1).

Restait à savoir comment Ki67 empêche la coalescence des chromosomes. Grâce à des expériences utilisant différentes versions fluorescentes de cette protéine, les auteurs montrent qu'un grand nombre de protéines Ki67 (270000) tapissent les chromosomes mitotiques: elles forment une sorte de «brosse » à poils longs d'environ $90 \mathrm{~nm}$ (approximativement l'épaisseur de la périphérie chromosomique [4]), denses (espacés en moyenne de $69 \mathrm{~nm}$, soit à peu près 210 molécules par $\mu \mathrm{m}^{2}$ ) et hautement chargés positivement sur toute leur longueur (Figure 2) [5]. Ki67, dont l'expression, au cours du cycle cellulaire, est maximale en mitose [6], est en effet une protéine géante et l'une des plus basiques du protéome humain [5]. Les auteurs montrent de plus que les protéines Ki67 sont orientées par leur caractère «amphiphile», leur domaine $\mathrm{C}$-terminal $\mathrm{LR}^{3}$ se liant au chromosome, et le reste de la protéine pointant vers le cytoplasme (Figure 2) [5]. Par des expériences de surexpression transitoire de Ki67 dans des cellules

\footnotetext{
${ }^{3}$ LR : domaine contenant 6 répétitions chez l'homme (5 chez la souris) de la paire leucine-arginine, deux acides aminés respectivement désignés, par convention, par les lettres $L$ et $R$.
} 
Cellule HeLa témoin

+ nocodazole
Cellule HeLa dépourvue de Ki67

(knock-out ou knock-down pour Ki67)

$$
\text { + nocodazole }
$$

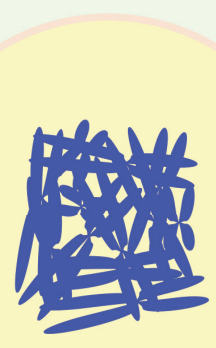

Cellule HeLa knock-out Ki67

+ Ki67 exogène surexprimée

+ nocodazole
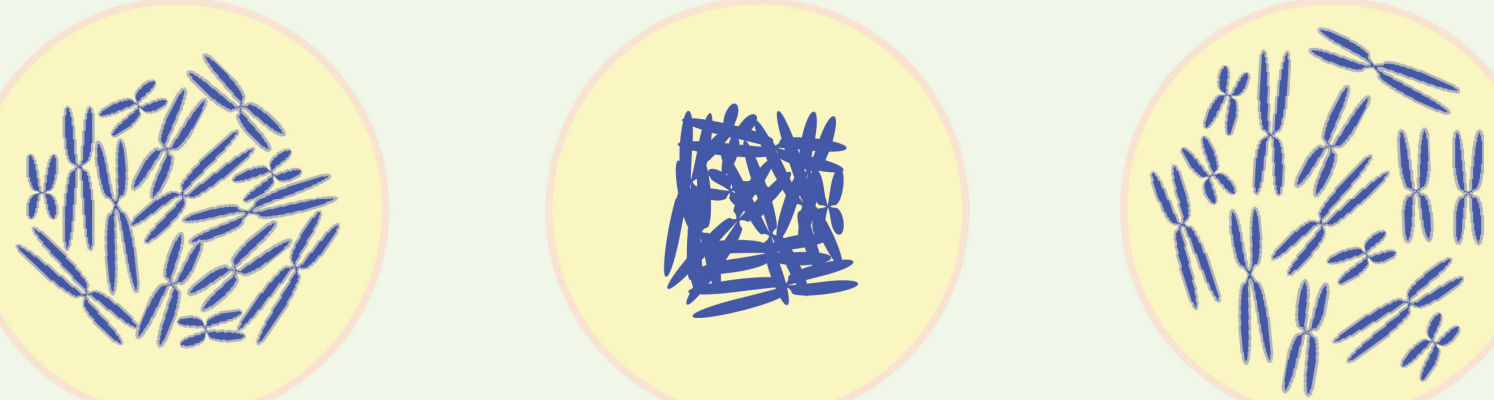

Figure 1. Ki67 est essentielle à la dispersion des chromosomes mitotiques. Dans des cellules HeLa traitées par le nocodazole (bloquées en prométaphase), les chromosomes mitotiques sont dispersés dans tout le cytoplasme et bien individualisés. En revanche, dans des cellules HeLa dépourvues de protéine Ki67 par knock-down ou knock-out de MK167 et traitées par le nocodazole, les chromosomes sont rassemblés en une masse unique et sont pratiquement immobiles. Inversement, un excès de Ki67 obtenu par surexpression transitoire provoque une dispersion accrue des chromosomes mitotiques (adapté de $[5,7]$ ).

dépourvues du gène $M K 167$, les auteurs montrent enfin que la distance entre les chromosomes augmente à mesure que la densité de Ki67 augmente [5] (Figure 1). Cet effet est dû à l'accroissement du nombre de charges positives et, probablement, à l'étirement corrélatif des protéines Ki67 perpendiculairement à l'axe des chromosomes [5]. La «brosse » Ki67 s'oppose donc à la coalescence des chromosomes par répulsion stérique et électrostatique [5]. Les auteurs concluent de l'ensemble de ces données structurales et fonctionnelles que Ki67 agit comme un surfactant naturel permettant la dispersion des chromosomes mitotiques [5].

Ces résultats, outre le fait qu'ils éclairent le rôle de Ki67 et celui de la périphérie chromosomique, permettent peut-être d'élucider quelques autres mystères. Par exemple, la protéine Ki67 n'existe que chez les vertébrés, et sa séquence diffère notablement entre espèces $[2,4,9,10]$, ce qui semble surprenant pour une protéine aussi étroitement associé à un phénomène universel comme la prolifération cellulaire. Les auteurs montrent que de nombreux mutants de Ki67 présentant des délétions, voire des inversions de différentes régions, peuvent, par surexpression transitoire, compenser plus ou moins efficacement une déficience du gène MKI67 : seul le domaine LR de Ki67 est nécessaire, mais pas suffisant, pour disperser les chromosomes mitotiques [5]. Les résultats indiquent que l'efficacité des mutants augmente avec leur taille et leur charge électrique totale [5]. La relative modestie de ces contraintes est en accord avec le fait que d'autres protéines chromosomiques riches en charges positives puissent, à condition d'être fortement surexprimées, disperser les chromosomes mitotiques [5]. Il existe donc, pour cette fonction, un certain degré de redondance possible entre Ki67 et d'autres protéines chromosomiques, degré positivement corrélé à la charge et, sans doute, la taille de ces protéines [5]. Cependant, d'autres fonctions de Ki67 dans le cycle cellulaire reposent sur des contraintes structurales distinctes [2-4]. Un autre type de redondance, à un niveau plus organisationnel, contribue par ailleurs à la capacité des cellules à supporter sans trop de dommages la coalescence des chromosomes provoquée par l'absence de Ki67. La coalescence empêche les microtubules du fuseau mitotique d'accéder aux kinétochores des chromosomes, ce qui devrait empêcher la mitose [5]. Cependant, les microtubules «profitent » d'une courte fenêtre temporelle avant la coalescence, lorsque les chromosomes sont encore séparés grâce à leur accrochage à l'enveloppe nucléaire, juste avant sa disparition: en l'absence de Ki67, la mitose est plus longue, mais n'est pas bloquée [5].

Ces résultats soulèvent aussi de nouvelles questions et hypothèses. Pourquoi seul le domaine LR interagit-il avec les chromosomes [7] ? En plus de la répulsion entre les charges positives [5], existe-t-il d'autres interactions favorisant le déploiement des protéines Ki67 perpendiculairement aux chromosomes? L'absence de Ki67 altère-t-elle aussi la structure ou la condensation des chromosomes mitotiques [4, 9]? De plus, si les protéines Ki67 entourent les chromosomes lors de la mitose, elles se concentrent dans le nucléole pendant la majeure partie de l'inter- 
1

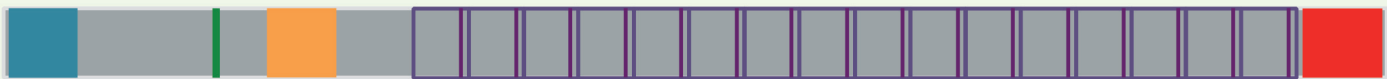

FRHA

PP1 CD

Répétition Ki67

LR

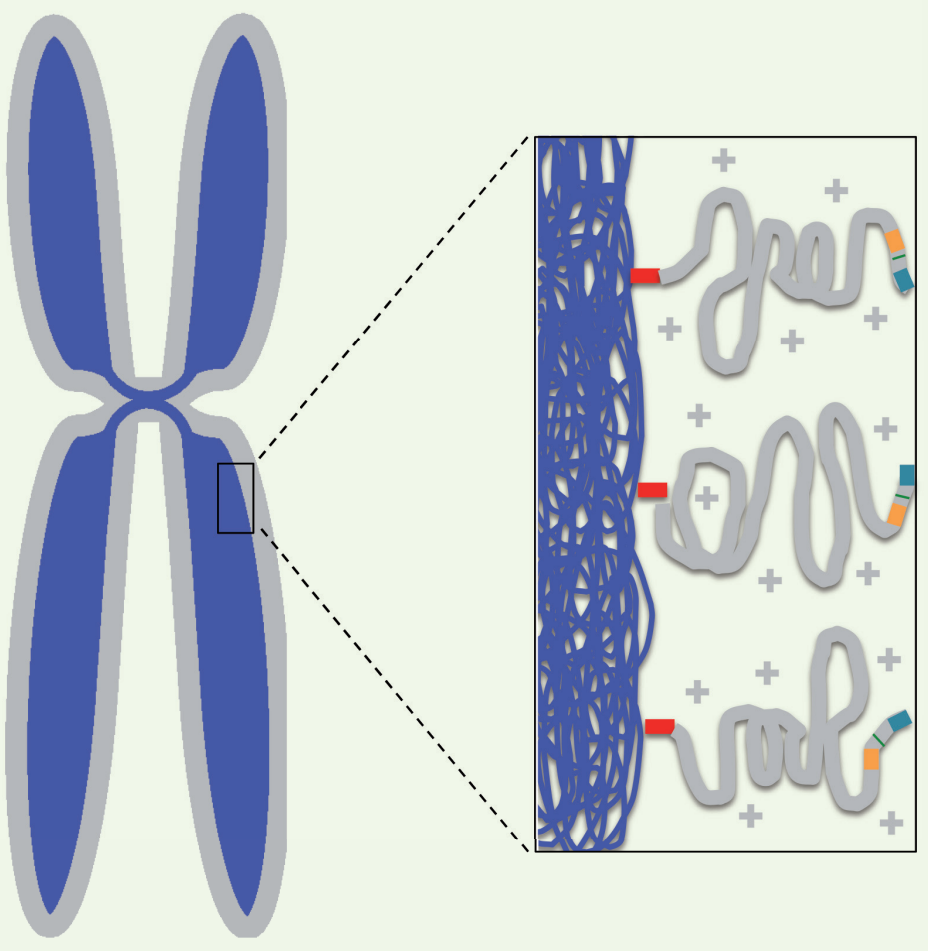

Figure 2. Ki67 présente les caractéristiques d'un surfactant naturel. Structure modulaire de la protéine Ki67. Le gène MKi67 code une très grande protéine, de 3256 résidus chez l'homme, constituée de plusieurs domaines fonctionnels. FHA : forkhead-associated domain, reconnaissant des phosphopeptides ; PPl : domaine de liaison à la protein phosphatase 1 ; CD : conserved domain; Répétitions Ki67 : 16 répétitions imparfaites de 122 résidus contenant un motif plus conservé de 22 résidus. LR : domaine contenant 6 répétitions de la paire leucine-arginine (deux acides aminés désignés par les lettres $L$ et $R$ ). Seule l'isoforme la plus longue est représentée [2] (adapté de [2, 9]). Les protéines Ki67 sont très chargées positivement et présentent une structure « amphiphile » : le domaine LR interagit avec la chromatine tandis que la protéine pointe vers le cytoplasme. Les protéines Ki67 forment ainsi une «brosse » assurant la dispersion des chromosomes par effet stérique et électrostatique (adapté de [5]).

phase $[2,4,5,7]$. Quelle est la fonction de Ki67 dans le nucléole? Un rôle de Ki67 dans l'organisation de l'hétérochromatine périnucléolaire a été rapporté $[2,4,10]$; les résultats résumés ici, et l'importance des phénomènes de séparation de phase dans la formation et l'organisation des nucléoles, suggèrent que Ki67 pourrait aussi jouer le rôle d'un surfactant dans cet organite $[2,5,7]$. Les nucléoles disparaissent pendant la mitose, et nombre de leurs constituants sont alors, comme Ki67, relocalisés dans

la périphérie chromosomique $[4,11,12](\rightarrow)$

$(\rightarrow)$ Voir la Synthèse de D. Hernandez-Verdun et $\varepsilon$. Louvet, $m / s n^{\circ} 1$, janvier 2004, page 37
Une hypothèse propose que ce transit assure la répartition et la réutilisation rapide de ces constituants dans les cellules filles [4, 11]. L'absence de Ki67 perturbe la localisation de toutes les protéines de la périphérie chromosomique testées et entraîne le partage inégal entre cellules filles d'au moins une protéine majeure du nucléole, la nucléoline [2, 4, 9-11]. La couche de protéines Ki67 autour des chromosomes joue donc aussi un rôle fondamental dans l'organisation de la périphérie chromosomique et sa fonction de transport «trans-mitotique »[4]. $\diamond$ Ki67: a surfactant of mitotic chromosomes

\section{LIENS D'INTÉRÊT}

Les auteurs déclarent n'avoir aucun lien d'intérêt concernant les données publiées dans cet article.

\section{RÉFÉRENCES}

1. Gerdes J, Schwab U, Lemke H, Stein H. Production of a mouse monoclonal antibody reactive with a human nuclear antigen associated with cell proliferation. Int J Cancer 1983; $31: 13-20$.

2. Sun X, Kaufman PD. Ki-67: more than a proliferation marker. Chromosoma 2018 ; 127 : 175-86.

3. Cidado J, Wong Hy, Rosen DM, et al. Ki-67 is required for maintenance of cancer stem cells but not cell proliferation. Oncotarget $2016 ; 7: 6281-93$.

4. Booth DG, Earnshaw WC. Ki-67 and the chromosome periphery compartment in mitosis. Trends Cell Biol 2017 ; 27 : 906-16

5. Cuylen S, Blaukopf C, Politi AZ, et al. Ki-67 acts as a biological surfactant to disperse mitotic chromosomes. Nature 2016 ; 535 : 308-12. 


\section{RÉFÉRENCES}

6. Sobecki M, Mrouj K, Colinge J, et al. Cell-cycle regulation accounts for variability in $\mathrm{Ki}-67$ expression levels. Cancer Res 2017 ; 77 : 2722-34.

7. Brangwynne CP, Marko JF. A sticky problem for chromosomes. Nature 2016 ; $535: 234-35$

8. Tremblay JP. Crispr, un système qui permet de corriger ou de modifier l'expression de gènes responsables de maladies héréditaires. Med Sci (Paris) 2015 ; 31 1014-22.

9. Takagi M, Natsume T, Kanemaki MT, Imamoto N. Perichromosomal protein Ki67 supports mitotic chromosome architecture. Genes Cells 2016 ; 21 : 1113-24.

10. Sobecki M, Mrouj K, Camasses A, et al. The cell proliferation antigen $\mathrm{Ki}-67$ organises heterochromatin. elife 2016 ; 5 : el3722.

\section{NOUVELLE}

Antoine Fruleux, Arezki Boudaoud
11. Booth DG, Takagi M, Sanchez-Pulido L, et al. Ki-67 is a PPl-interacting protein that organises the mitotic chromosome periphery. elife 2014 ; 3 : e01641.

12. Hernandez-Verdun $D$, Louvet $\varepsilon$. Le nucléole : structure, fonctions et maladies associées. Med Sci (Paris) $2004 ; 20: 37-44$.

\section{Les forces qui maintiennent l'ordre parmi les cellules}

> La majorité des animaux, dont les humains, ont une symétrie bilatérale. Ainsi, en général, nos deux mains se superposent quasi parfaitement, et si nous étendons nos bras, nous trouvons que leurs longueurs sont quasiment identiques. De même, un plant de pétunia présente des fleurs dont les formes et tailles sont quasi identiques. II peut sembler banal que deux organes qui résultent d'un même processus de développement soient identiques comme le sont deux pièces usinées sortant d'une même chaîne de production, mais c'est faire abstraction du fait que chaque organe est composé d'une myriade de cellules qui croissent, se divisent... et se comportent individuellement de manière aléatoire, comme le montre une carte de croissance cellulaire (Figure 1). Se pose ainsi la question de savoir comment des organes ou des organismes atteignent une taille et une forme bien précises au terme de leur développement, alors que la croissance des cellules qui les composent fluctue dans le temps et dans l'espace.

Répondre à cette question nécessite de considérer les effets statistiques liés au grand nombre de cellules qui constituent un organe. Au fur et à mesure que l'organe croît et que ses cellules prolifèrent, les fluctuations individuelles des cel- lules ont de moins en moins d'impact sur celle de l'organe et, par conséquence, l'incertitude sur la taille de l'organe décroît (ou la précision de la taille augmente). De manière comparable, dans une école, la proportion de filles et de garçons dans des groupes pris au hasard fluctue entre les groupes, mais les fluctuations décroissent avec la taille du groupe, de sorte que le rapport garçons/ filles se rapproche de 1,06 à l'échelle de l'école. C'est la loi des grands nombres. Si les cellules d'un organe grandissaient de manière aléatoire et indépendante, la croissance de l'organe, qui intègre la croissance de toutes les cellules, deviendrait de moins en moins variable quand le nombre de cellules augmente [1]. Cependant, un tel scénario ne rend pas vraiment compte de la réalité, car les cellules communiquent entre elles et ne sont donc pas toutes indépendantes les unes des autres. C'est ainsi que nous nous sommes intéressés aux propriétés statistiques émergentes des tissus pour comprendre comment les organes peuvent se développer avec une forme et une taille reproductibles [2].

Outre ces effets statistiques, il convient d'identifier et de prendre en compte les mécanismes à l'œuvre pour que des organes atteignent des formes reproductibles. Si les cellules d'un tissu ont
Reproduction et développement des plantes, Université de Lyon, École normale supérieure de Lyon, Université Claude Bernard Lyon 1, INRA, CNRS, 46 allée d'Italie, 69364 Lyon Cedex 07, France. arezki.boudaoud@ens-lyon.fr

une croissance partiellement aléatoire, les cellules qui grandissent le plus vite ont tendance à pousser leurs voisines. $\varepsilon n$ résultent des forces au sein du tissu. De manière générale, la croissance non uniforme des cellules suscite des forces (contrainte mécanique interne) dans le tissu. De telles forces pourraient être perçues et utilisées par la cellule [3]. La situation ressemble à celle d'une ronde dont les danseurs ont les yeux bandés: chaque danseur a son propre rythme et le rythme fluctue d'un danseur à l'autre; chaque danseur perçoit donc des forces transmises de main à main par les danseurs voisins. Les danseurs pourraient-ils utiliser ces forces pour adapter leur rythme?

De nombreux travaux de recherche ont montré que les cellules ont la capacité de percevoir les forces mécaniques et d'y répondre activement de différentes manières. Il a été proposé que le taux de prolifération cellulaire augmente ou diminue lorsque les cellules sont soumises respectivement à des forces de tension ou de compression [3], et cette hypothèse s'est révélée pertinente pour le développement de la mouche du vinaigre (drosophile) et du poisson-zèbre [4-6]. La réponse aux forces mécaniques peut aussi se traduire par une rigidification du tissu, 